\title{
Evolution of Low-Mass Helium Dwarfs in Interacting Binaries:
}

\section{Application to $4 \mathrm{U} 1820-30$}

\author{
Lorne A. Nelson \\ CITA, University of Toronto, Toronto, Ontario, Canada \\ Paul C. Joss and Saul Rappaport \\ Center for Theoretical Physics, MIT, Cambridge, Mass., USA
}

\section{Introduction}

A number of evolutionary scenarios have been proposed that lead to the formation of binary systems consisting of a degenerate dwarf in orbit with a neutron star. In fact, most degenerate dwarfs in close-binary systems are probably the cores of evolved stars whose envelopes have been stripped by one or more episodes of common-envelope evolution. Once the envelope has been removed, the newly exposed core of the giant will have an effective temperature and radius that are considerably larger than the corresponding quantities in an older, highly degenerate dwarf. It is important for at least two reasons to evaluate the subsequent cooling history of such objects: (i) For detached binaries, we want to be able to infer from the current effective temperature and luminosity of the degenerate dwarf the elapsed time since the core of its progenitor giant was exposed. (ii) For semi-detached binaries, we must know the evolution of the radius of the degenerate dwarf in order to compute important relationships among the orbital period and its rate of change, the mass of the degenerate dwarf, and the mass-transfer rate.

One example of a system for which this type of analysis can be applied is the globular cluster X-ray burst source 4U1820-30. Recently, Stella, Priedhorsky, and White (1987) discovered a stable $685 \mathrm{~s}$ periodicity in the $\mathrm{X}$-ray flux from this source. Using archival satellite data dating back to 1976, Morgan, Remillard, and Garcia (1988) confirmed the 685 s periodicity and set an upper limit on the fractional rate of change in the period of $\dot{P} / P<2.7 \times 10^{-7} \mathrm{yr}^{-1}$. This high degree of stability provides convincing evidence that the periodic modulation in the $\mathrm{X}$-ray intensity results from binary orbital motion. If the orbital period of $4 \mathrm{U} 1820-30$ is indeed $685 \mathrm{~s}$, then it is the most compact binary yet discovered. Based on the recurrence times of the type I X-ray bursts that are observed and given our understanding of these events as thermonuclear flashes on neutron stars (see, e.g., Joss 1978), we can conclude that this system contains a neutron star that is accreting helium-rich matter. Moreover, the mean density of the Roche lobe-filling companion star can be inferred from the orbital period to be $\sim 3100 \mathrm{~g} \mathrm{~cm}^{-3}$. This density is indicative of a low-mass dwarf $\left(\lesssim 0.1 M_{\odot}\right)$ that is at least partially degenerate.

\section{Evolution of the Semi-Detached Binary}

In order to examine the present evolutionary status of 4 U1820-30, we assume that it is a semi-detached binary system consisting of a neutron star of mass $M_{1}$ in a circular orbit with a low-mass secondary of mass $M_{2}$. Systemic angular momentum losses drive the mass transfer and can include (1) gravitational radiation losses as specified by the Einstein quadrupole formula $\left(\dot{J}_{G R}<0\right)$, and $(2)$ angular momentum that is carried away by any matter that may be ejected from the binary system. By including both of these possibilities, a general expression for the 
mass-transfer rate $\dot{M}_{2}$ was derived by Rappaport, Verbunt, and Joss (1983):

$$
-\frac{\dot{M}_{2}}{M_{2}}=\frac{\frac{1}{2} \dot{R}_{T H} / R_{2}-\dot{J}_{G R} / J}{\left[\left(5 / 6+\xi_{a d} / 2\right)-\frac{(1-\beta)}{3(1+q)}-\frac{(1-\beta) \alpha(1+q)+\beta}{q}\right]} .
$$

Here $\left(\dot{R}_{T H} / R_{2}\right)^{-1}$ is the thermal contraction time scale of the secondary ( $R_{2}$ being the radius of the secondary), $J$ is the orbital angular momentum, $\xi_{a d}$ is the "adiabatic stellar index" (i.e., $\xi_{a d}=$ $d \ln R_{2} / d \ln M_{2}$ evaluated at constant internal specific entropy), and $q \equiv M_{1} / M_{2}$. The parameters $\alpha$ and $\beta$ describe angular momentum losses due to possible systemic mass loss (see Rappaport $e t$ al. 1983 for details). Since the secondary must fill its Roche lobe, the orbital period can be related to the secondary's mass and radius by the well-known relation $P=9 \pi(2 G)^{-1 / 2} R_{2}^{3 / 2} M_{2}^{-1 / 2}$. Thus the fractional rate of change in the orbital period can be expressed as

$$
\frac{\dot{P}}{P}=-\frac{1}{2} \frac{\dot{M}_{2}}{M_{2}}+\frac{3}{2} \frac{\dot{R}_{2}}{R_{2}}
$$

Equations (1) and (2) can be integrated to obtain the systemic evolution once the response of the secondary to mass loss has been ascertained. For the low-mass secondaries of interest here, the thermal contraction time scale is generally much larger than the gravitational radiation time scale (see $\S 3$ ), so that the star responds almost adiabatically. Hence, $\xi_{\text {ad }}$ can be determined to a good approximation from the mass-radius relationship for zero-temperature stars. Specifically, we have taken the radius of the secondary to be $R_{2}=f R_{Z S}\left(M_{2} ; X, Z\right)$, where $R_{Z S}$ is the radjus of a zero-temperature star as defined by Zapolsky and Salpeter (1969) and $X$ and $Z$ are the standard composition parameters for the secondary (which we have assumed to be chemically homogeneous). The factor $f(\geq 1)$ accounts for thermal contributions to the equation of state and is assumed to be constant in any given evolutionary calculation (see §III). We note that while the inferred values of $\xi_{a d}$ may not be highly accurate when $f$ exceeds unity, our results are rather insensitive to small variations in $\xi_{\text {ad }}$.

\section{Cooling Evolution of Helium Dwarfs}

We have determined the thermal contraction time scales of low-mass $\left(\lesssim 0.2 M_{\odot}\right)$ helium dwarfs by calculating their evolutionary cooling histories. Very little previous work has been carried out on the cooling of low-mass helium or CO degenerate dwarfs (but see D'Antona, Magni, and Mazzitelli 1972 for a detailed study of the evolution of a $0.15 M_{\odot}$ helium dwarf). We are particularly interested in determining at what point during the evolution the thermal contraction time scale, $\tau_{T H} \equiv R_{2} / \dot{R}_{T H}$, becomes longer than the gravitational radiation time scale, $\tau_{G R} \equiv J / \dot{J}_{G R}$. For all times during the binary evolution when $\tau_{T H}>>\tau_{G R}$, we are justified in neglecting the $\dot{R}_{T H} / R_{2}$ term in equation (1). It can also be shown that $f \simeq$ constant when this criterion is satisfied. In this way, the thermal evolution is decoupled from the mass-transfer evolution.

We have followed the cooling of the degenerate dwarf using a version of a Henyey-type code that has previously been used to study the evolution of very low-mass stars in cataclysmic variables (see Nelson, Chau, and Rosenblum 1985 for further details). We incorporated extensive improvements to the evaluation of the equation of state and the radiative and conductive opacities. Specifically, we implemented the Magni and Mazzitelli (1979) description of the equation of 
state for pure helium. The radiative and electron conduction opacity coefficients were interpolated from the Cox-Stewart (1970) and Hubbard and Lampe (1969) opacity tables, respectively. Extrapolations beyond the limits of the opacity tables were sometimes required but usually occurred in regions of the star that were unstable against efficient convection, so that the numerical accuracy of the opacities was not overly important. Since 4U1820-30 is located in the globular cluster NGC 6624 , for which the metallicity $Z$ is reasonably well known, we adopted the observationally inferred value $(Z \simeq 0.003$ ). We did not attempt to include the effects of chemical diffusion in this study, since they are thought to be unimportant (Alcock and Illarianov 1982).

In Figure 1 we compare the magnitudes of the thermal contraction and gravitational radiation time scales for three representative stellar masses as functions of $f$ (the ratio of the stellar radius to the radius of a completely degenerate star of the same mass and composition). We have taken the mass of the neutron-star primary to be $1.4 M_{\odot}$. As can be seen in the figure, the thermal contraction time scale is always much longer than the gravitational radiation time scale for $f \lesssim 1.8$. Values of $f$ larger than $\sim 1.8$ correspond to stellar ages of less than $\sim 3 \times 10^{8}$ yr. Hence, we can reasonably conclude that if the progenitor binary (after an assumed phase of common-envelope evolution) took at least $3 \times 10^{8} \mathrm{yr}$ to enter into a semi-detached state, then we are justified in neglecting the $\dot{R}_{T H} / R_{2}$ term in equation (1) and in taking $f$ to be constant. We also find that none of our models underwent crystallization, even at late ages $\left(\sim 10^{10} \mathrm{yr}\right)$.

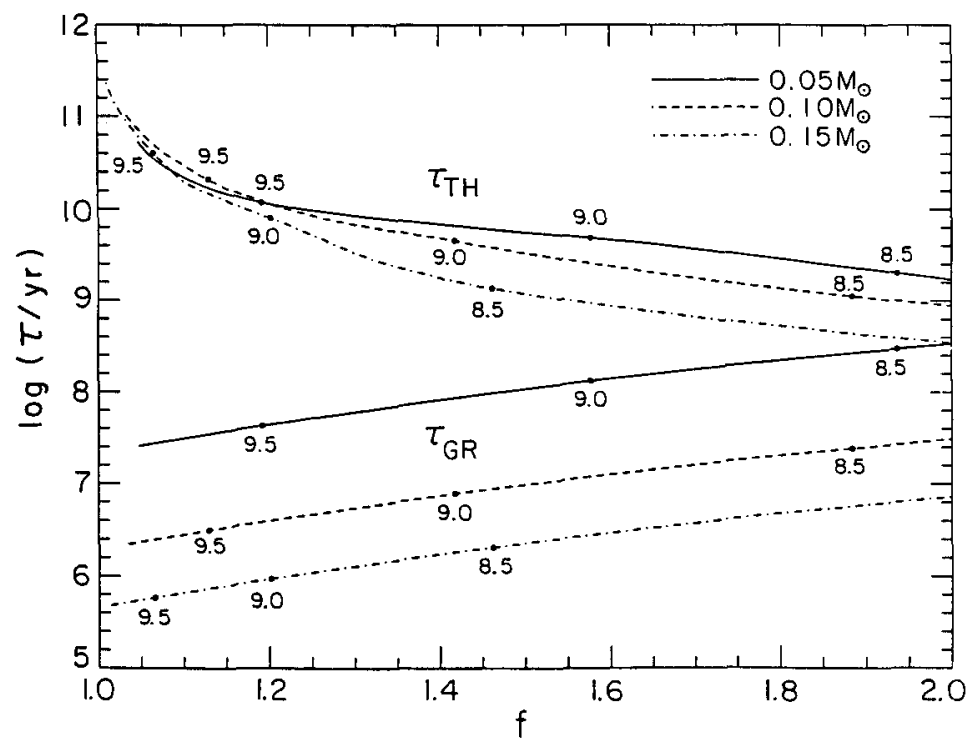

Figure 1. Calculated thermal evolution of a low-mass helium degenerate dwarf. The upper set of curves shows the thermal time scale, $\tau_{T H}$, as a function of $f$ (the ratio of the stellar radius to the radius of a completely degenerate star of the same mass and composition). The legend indicates which stellar mass has been used in each calculation. The numbers next to the heavy dots on the curves are the logarithms of the elapsed evolutionary times. The lower set of curves shows, for comparison, the gravitational-radiation time scale, $\tau_{G R}$, of a semi-detached binary as a function of $f$, under the assumptions that the helium star is the lobe-filling secondary and that the primary has a mass of $1.4 M_{\odot}$ (see text). 


\section{Discussion}

Under the assumptions that the $4 \mathrm{U} 1820-30$ system consists of a neutron star with a mass of $1.4 M_{\odot}$ and a radius of $10 \mathrm{~km}$ in a 685 -s orbit with a fully degenerate $(f=1)$, helium-dwarf companion, and that no mass is being lost from the system during the mass-transfer process, it is possible to derive $M_{2}, \dot{P} / P$, and the observed X-ray luminosity $L_{x}$. The nominally predicted values for the system observables are $\dot{P} / P \simeq 1.1 \times 10^{-7} \mathrm{yr}^{-1}, L_{x} \simeq 8 \times 10^{37} \mathrm{ergs} \mathrm{s}^{-1}$, and $M_{2} \gtrsim 0.057 M_{\odot}$. The calculated value of $\dot{P} / P$ is entirely consistent with the observed upper limit (see $\S 1$ ), and the calculated value of $L_{x}$ is just consistent with the high end of the range of measured values of $L_{x}$. We conclude that the measured and inferred properties of the 4U1820-30 system can be readily accounted for in an evolutionary scenario that invokes conservative mass transfer driven by the emission of gravitational radiation. Since the measured upper limit on $\dot{P} / P$ is only about a factor of two higher than the value predicted for our standard model, we expect that the rate of change of the orbital period will be detectable within the next few years. Hence, $4 \mathrm{U} 1820-30$ could be the first mass-transfer system where the rate of change of orbital period resulting from gravitational radiation reaction can be measured directly.

We can also utilize the observationally inferred range of values for $L_{\boldsymbol{x}}$ and the upper limit on $\dot{P} / P$ to set quantitative constraints on some of the system parameters. In particular, we find that for $M_{1}=1.4 M_{\odot}$ and conservative mass-transfer, $f$ must be no greater than $\sim 1.2$. Assuming that the initial value of $M_{2}$ is $\leq 0.1 M_{\odot}$, and given that $f$ is approximately constant during the mass-transfer evolution, we see from Fig. 1 that at least $\sim 3 \times 10^{9}$ yr must have elapsed between the formation of the dwarf-star/neutron-star binary and the start of the mass-transfer phase (i.e., during the detached phase). (If the secondary retained a thin hydrogen-rich envelope after the spiral-in phase, the cooling time would only be increased.) Thus, this system might well have formed at a much earlier epoch in the history of the globular cluster NGC 6624, when the masses of the cluster giants would have been substantially higher than at the present time.

An earlier version of portions of this paper was published by Rappaport et al. (1987). This work was supported in part by the National Science Foundation under grant AST-8419834, by the National Aeronautics and Space Administration under grant NSG-7643, and by the Natural Sciences and Engineering Research Council (NSERC) of Canada.

\section{References}

Alcock, C., and Illarionov, A. 1980, Ap. J., 235, 534.

Cox, A. N., and Stewart, J. N. 1970, Ap. J. Suppl., 19, 243.

D'Antona, F., Magni, G., and Mazzitelli, I. 1972, Astrophys. Sp. Sci., 19, 151.

Hubbard, W. B., and Lampe, M. 1969, Ap. J. Suppl., 18, 297.

Joss, P. C. 1978, Ap. J. (Letters), 225, L123.

Magni, G., and Mazzitelli, I. 1979, Astr. Ap., 72, 134.

Morgan, E. H., Remillard, R. A., and Garcia, M. R. 1988, Ap. J., 324, 851.

Nelson, L. A., Chau, W. Y., and Rosenblum, A. 1985, Ap. J., 299, 658.

Rappaport, S., Nelson, L. A., Ma, C. P., and Joss, P. C. 1987, Ap. J., 322, 842.

Rappaport, S., Verbunt, F., and Joss, P. C. 1983, Ap. J., 275, 713.

Stella, L., Priedhorsky, W., and White, N. E. 1987, Ap. J. (Letters), 312, L17.

Zapolsky, H. S., and Salpeter, E. E. 1969, Ap. J., 158, 809. 